\title{
Histological Examination of the Gadolinium-enhanced Dura Mater Around Meningiomas on Magnetic Resonance Imaging
}

\author{
Keiichi SaKaI, Tsuyoshi TADA, Kazuaki FuKASAKU, Kazuhiko KYoshima \\ and Shigeaki KOBAYASHI
}

Department of Neurosurgery, Shinshu University School of Medicine, Matsumoto, Nagano

\begin{abstract}
Magnetic resonance imaging often demonstrates gadolinium (Gd) enhancement of the dura mater around meningiomas. The $\mathrm{Gd}$-enhanced dura mater was histologically investigated to detect meningioma cells. Gd enhancement of the dura mater occurred in $11(79 \%)$ of the 14 meningiomas studied, and extended as far as $35 \mathrm{~mm}$ from the tumor. Histological examination revealed generation of vascular-rich loose connective tissue at the surface of the dura in all five tumors examined. Some clusters of meningothelial cells were distributed in the loose connective tissue in three of the five specimens, and one cluster was obviously neoplastic. These observations suggest that clusters of meningioma cells occur in the Gd-enhanced dura mater around meningiomas.
\end{abstract}

Key words: dura mater, gadolinium, magnetic resonance imaging, meningioma, grading systems

\section{Introduction}

Meningiomas are well-demarcated benign tumors, but the frequency of recurrence is unexpectedly high, even after total tumor removal. ${ }^{6}$ The reason is unclear, although regional multicentricity of meningiomas has been suggested. ${ }^{2,3)}$

Recently, magnetic resonance (MR) imaging has demonstrated gadolinium (Gd) enhancement of the dura mater surrounding meningiomas, known as the "meningeal sign,", (0) "flare sign,",1) or "dural tail,",4) which has become one of the diagnostic characteristics of meningioma. ${ }^{4,(4)}$ Here, we report our histological investigation of surgical specimens of the dura mater showing the "meningeal flare sign" to detect the inclusion of meningioma cells.

\section{Materials and Methods}

This study included 14 patients with meningioma who were surgically treated at Shinshu University Hospital. All underwent MR imaging with Gd enhancement, computed tomography (CT) with con-

Received April 30, 1992; Accepted December 8, 1992 trast medium, and cerebral angiography preoperatively. There were 12 females and two males aged between 13 and 64 years (mean 49.6 yrs). All tumors were removed totally or subtotally. In five patients, the Gd-enhanced dural strips surrounding the tumor could be resected radically. Serial sections of the resected dura were stained with $\mathrm{HE}$, and immunohistochemically with anti-vimentin monoclonal antibody (Dako Corp., Santa Barbara, Cal, U.S.A.) ${ }^{11}$ and anti-epithelial membrane antigen (EMA) monoclonal antibody (Dako), ${ }^{7,9,13)}$ to detect the presence of meningothelial cells in the dura mater.

\section{Results}

Table 1 summarizes the 14 patients in this study. The tumor histological subtypes were 12 meningotheliomatous, one transitional, and one angioblastic meningiomas. MR imaging Gd enhancement around meningiomas was seen in 11 cases $(79 \%)$ in all subtypes. The area of Gd enhancement of the dura mater extended as far as $35 \mathrm{~mm}$ (average $21.1 \mathrm{~mm}$ ) from the attachment of the tumor. The thickness of the Gd-enhanced dura mater varied from normal to apparently abnormal continuous with the tumor. 
Table 1 Summary of 14 patients with meningioma

\begin{tabular}{|c|c|c|c|c|c|c|}
\hline $\begin{array}{l}\text { Case } \\
\text { No. }\end{array}$ & $\begin{array}{l}\text { Age } / \\
\text { Sex }\end{array}$ & Location of tumor & Tumor size (mm) & Histological subtype & $\begin{array}{c}\text { Meningeal } \\
\text { enhancement }\end{array}$ & $\begin{array}{l}\text { Maximal width } \\
(\mathrm{mm})^{* *}\end{array}$ \\
\hline 1 & $47 / \mathrm{F}$ & tentorial & $30 \times 20 \times 30$ & meningotheliomatous & yes & 15 \\
\hline 2 & $64 / F$ & falx & $40 \times 50 \times 40$ & meningotheliomatous & yes & 20 \\
\hline 3 & $61 / \mathrm{F}$ & skull base & $60 \times 50 \times 45$ & meningotheliomatous & yes & 18 \\
\hline 4 & $55 / \mathrm{F}$ & tentorial & $20 \times 15 \times 50$ & meningotheliomatous & yes & 28 \\
\hline 5 & $60 / \mathrm{F}$ & sphenoid ridge & $30 \times 30 \times 20$ & meningotheliomatous & yes & 20 \\
\hline $6^{*}$ & $13 / \mathrm{F}$ & sphenoid ridge & $30 \times 25 \times 30$ & meningotheliomatous & yes & 35 \\
\hline 7 & $64 / F$ & skull base & $90 \times 80 \times 100$ & meningotheliomatous & yes & 35 \\
\hline $8^{*}$ & $44 / \mathrm{M}$ & sphenoid ridge & $30 \times 30 \times 30$ & meningotheliomatous & yes & 12 \\
\hline $9^{*}$ & $50 / \mathrm{F}$ & falx & $55 \times 80 \times 65$ & transitional & yes & 10 \\
\hline $10^{*}$ & $52 / \mathrm{M}$ & falx & $50 \times 45 \times 50$ & angioblastic & yes & 15 \\
\hline $11^{*}$ & $41 / F$ & sphenoid ridge & $25 \times 15 \times 25$ & meningotheliomatous & yes & 24 \\
\hline 12 & $50 / \mathrm{F}$ & olfactory groove & $55 \times 65 \times 45$ & meningotheliomatous & no & - \\
\hline 13 & $52 / \mathrm{F}$ & posterior fossa & $50 \times 50 \times 50$ & meningotheliomatous & no & - \\
\hline 14 & $42 / F$ & tentorial & $20 \times 8 \times 15$ & meningotheliomatous & no & - \\
\hline
\end{tabular}

*Dura mater around meningioma examined histologically. ${ }^{* *}$ Maximal width of the Gd-enhanced dura mater around the meningioma.

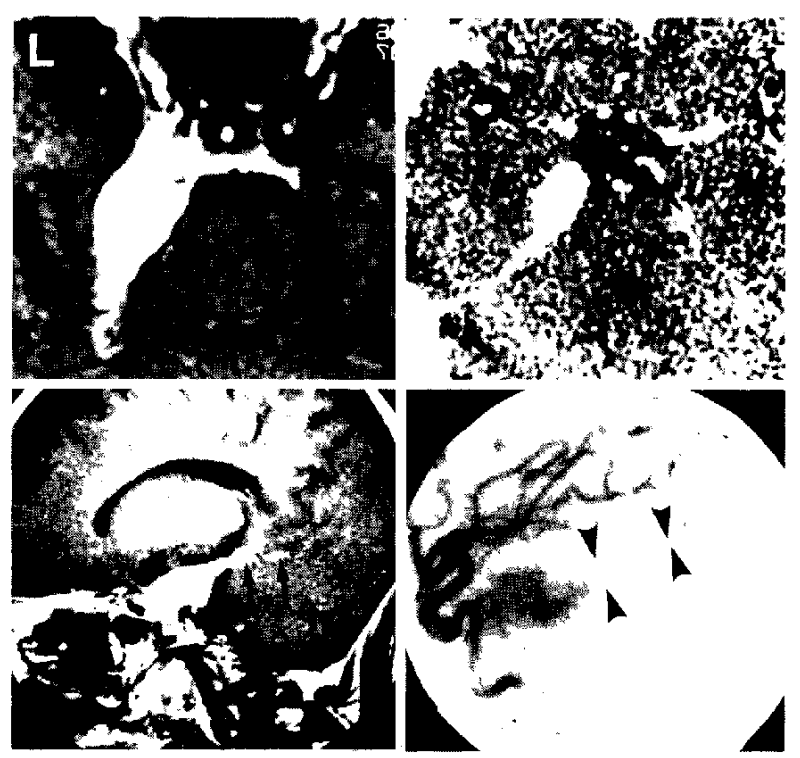

Fig. 1 Tentorial meningioma in Case 4. upper left: Axial Gd-enhanced $T_{1}$-weighted $M R$ image, showing the enhanced thick tentorium continuous with the tumor mass. upper right: Axial postcontrast CT scan, showing the enhanced thick tentorium. lower left: Sagittal Gd-enhanced $\mathrm{T}_{1}$-weighted MR image, showing the enhanced tentorium continuous with the tumor mass (arrows). lower right: Angiogram, showing tumor staining at the tentorial portion (arrowheads).
Only one patient (Case 4) revealed contrast enhancement on both CT scans and MR images at the same portion of the dura mater, and had the thickest Gd-enhanced dura. Angiograms revealed abnormal vessels in the same region (Fig. 1). These findings suggested that the Gd-enhanced dural portion corresponded to direct invasion by the tumor along the dura mater. CT scans showed no contrast enhancement of the dura mater in the remaining 10 cases.

Histological examination of the surrounding dura mater (Case 4 not included) revealed: 1) loose connective tissue containing numerous enlarged capillary vessels in the surface of the dura in all cases, and 2) various-sized clusters of meningotheliomatous cells in the loose connective tissue in three. Clusters of meningothelial cells were better visualized with immunohistochemical staining for EMA and vimentin.

\section{Illustrative Cases}

Case 6: A 13-year-old girl presented with medulloblastoma in the cerebellum at age 1 year. The tumor disappeared after radiation therapy following partial removal. Follow-up MR images revealed a newly developed mass at the right sphenoid ridge without local recurrence of the medulloblastoma (Fig. 2 left). The sphenoid ridge tumor removed was meningotheliomatous meningioma. Histological examination of the dural portion around the tumor insertion revealed numerous small clusters of meningothelial cells along the loose connective tissue in 
the dura (Fig. 2 right). The clusters were distributed diffusely in the loose connective tissue as far as the margin of the Gd-enhanced dura mater, $35 \mathrm{~mm}$ distant from the tumor attachment, and stained positively for vimentin and EMA.

Case 10: A 52-year-old male had a single epileptic seizure without neurological deficits. CT scans and MR images revealed a falx tumor in the left frontal lobe. The dura mater was well enhanced around the tumor (Fig. 3 left). The histological diagnosis of the tumor was angioblastic meningioma. Histological examination of the Gd-enhanced dura mater found vascular-rich loose connective tissue generating at the dura mater and one small cluster of meningothelial cells in the loose connective tissue of the dura $9 \mathrm{~mm}$ distant from the tumor attachment (Fig. 3 right); the cluster stained positively to antivimentin antibody.

Case 11: A 41-year-old female underwent surgery for a right sphenoid ridge meningioma, histologically diagnosed as meningotheliomatous meningioma. Three years later, she was readmitted due to regrowth of the residual tumor. Gd-enhanced MR images revealed enhancement of the surrounding dura extending to the temporal base $24 \mathrm{~mm}$ distant from the tumor attachment (Fig. 4 left). Operation discovered a solitary small mass at the margin of the Gd-enhanced dura. The regrown tumor with surrounding dura mater including the solitary mass was removed. Histological examination of the Gdenhanced dura mater revealed that the solitary small mass was located in loose connective tissue of the dura mater and was positive to anti-vimentin and anti-EMA antibodies (Fig. 4 center, right). The small mass was diagnosed as a newly developed meningotheliomatous meningioma.

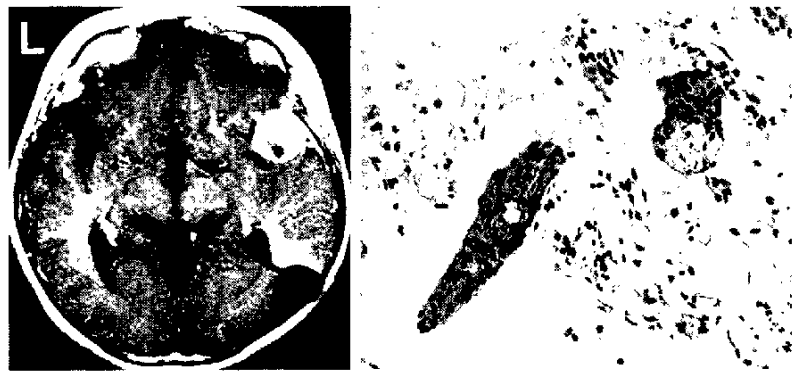

Fig. 2 Sphenoid ridge meningioma in Case 6 . left: Axial Gd-enhanced $T_{1}$-weighted MR image, showing the well-enhanced dura mater around the meningioma. right: Photomicrograph of the Gd-enhanced dura mater, containing numerous clusters of meningothelial cells only in the vascular-rich loose connective tissue on the surface. These clusters were within $35 \mathrm{~mm}$ of the tumor. HE stain, $\times 175$.
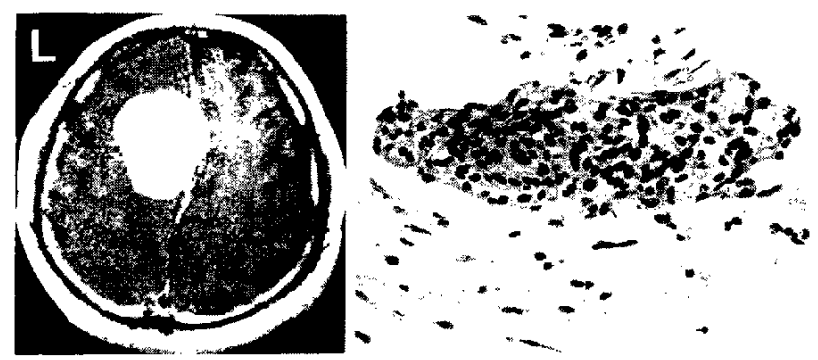

Fig. 3 Falx meningioma in Case 10. left: Axial Gdenhanced $T_{1}$-weighted MR image, showing the enhanced dura mater around the meningioma. right: Photomicrograph of the Gd-enhanced dura, showing a cluster of meningioma in the loose connective tissue. The cluster was $9 \mathrm{~mm}$ from the tumor attachment. HE stain, $\times 175$.
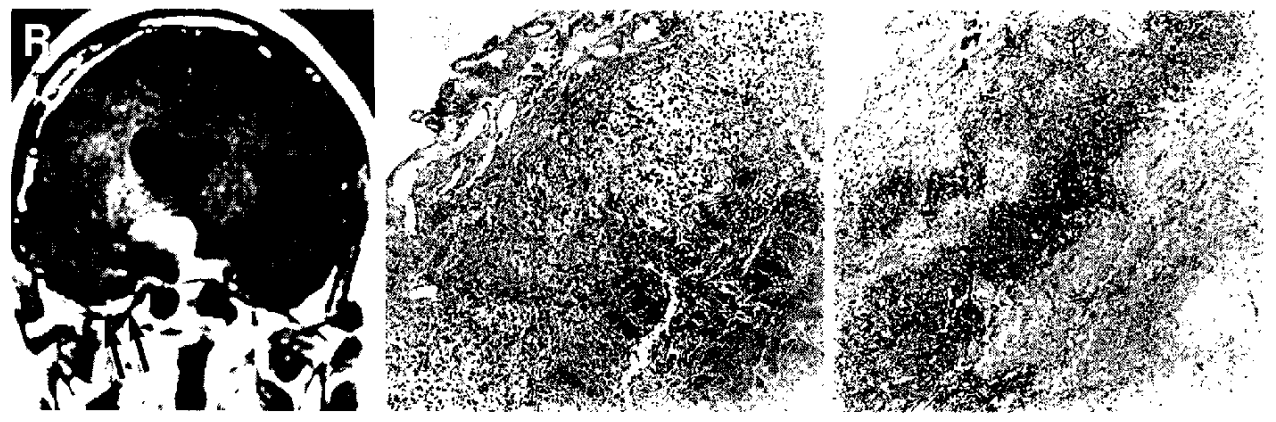

Fig. 4 Sphenoid ridge meningioma in Case 11. left: Coronal Gd-enhanced $T_{1}$-weighted MR image, showing the enhanced dura at the right temporal base (arrows). The signal loss at the tumor insertion is an artifact due to a hemostatic clip. center, right: Photomicrographs of serial sections of the dura, showing a small meningioma in loose connective tissue. center: HE stain, $\times 56$; right: EMA, $\times 56$. 


\section{Discussion}

Explanations for the Gd enhancement of the dura mater around meningiomas include tumor extension and loose connective tissue generation in the dura mater. Histological investigation has shown that the Gd-enhanced thickened dura corresponds to tumor extension within or around the dura. ${ }^{16)}$ In our series, Case 4 with the thickest dural enhancement on MR images was also enhanced on CT scans, and the angiograms showed abnormal staining continuous with the tumor mass. Our radiological diagnosis was tumor extension along the dura mater. In contrast, Aoki et al. ${ }^{1)}$ observed proliferation of loose connective tissue with many vessels along the Gd-enhanced dura. We also found vascular-rich loose connective tissue in the Gd-enhanced dura mater in all five cases examined. This would account for the Gd enhancement of the dura mater on MR images, especially when thin and without contrast enhancement on CT scans.

Nomura et $a l .{ }^{8)}$ reported that meningeal enhancement, possibly resulting from the proliferating capillaries of dura mater, occurred in approximately one third of cases following craniotomy. The interval between surgery and the appearance of the meningeal enhancement ranged from 4 days to 88 weeks. In our study, Cases 6 and 11 had received a previous craniotomy. However, MR images 12 years after craniotomy in Case 6 and 3 years in Case 11 showed dural enhancement for the first time. Furthermore, histological examination of the dural specimens revealed small clusters of meningothelial cells in the dura adjacent to sphenoid ridge meningioma in Case 6 and meningotheliomatous meningioma in Case 11. Therefore, the meningeal enhancement in our cases was not a reactive response to craniotomy.

Meningotheliomatous cells in the dura mater adjacent to the intracranial meningiomas corresponding to $\mathrm{Gd}$ enhancement has rarely been reported..$^{51} \mathrm{Ac}$ cording to Aoki et al., ") there is no tumor infiltration in the Gd-enhanced dura, while Tokumaru et al. ${ }^{15}$ observed clusters of tumor cells only within $1 \mathrm{~mm}$ of the tumor margin. In our study, clusters of meningothelial cells were distributed widely in the vascular-rich loose connective tissue corresponding to the area of Gd-enhanced dura mater. Especially in Case 11 , the meningothelial cells of the small meningioma were clearly neoplastic, and the smail tumor was located in the loose connective tissue of the Gd-enhanced dura mater. This suggests that clusters of meningothelial cells in the loose connective tissue can become neoplastic. We think that clus- ters of meningothelial cells in the Gd-enhanced dura mater around meningiomas is a cause of meningioma recurrence after Simpson's grade 1 surgery ${ }^{12}$ is achieved.

Borovich et $a l^{2,3)}$ reported that the neoplastic field can extend about $4 \mathrm{~cm}$ around the tumor margin in the form of macroscopic dural nodules and microscopic cell clusters. They proposed a supplementary grade to the Simpson's surgical grading ${ }^{12}$ : grade 0 , the operation entailing a wide resection of the dura around the attachment zone of the meningioma. Our observations show that the maximum extension of Gd-enhanced dura mater around meningiomas was about $35 \mathrm{~mm}$, and small clusters of meningothelial cells occurred in the marginal zone of the enhanced dura. Therefore, the Gd-enhanced dura mater around meningiomas shown by MR imaging, even if not enhanced on CT scans, reflect the existence of vascular-rich loose connective tissue at the surface of the dura mater, where clusters of meningioma cells may be present.

\section{References}

1) Aoki S, Sasaki Y, Machida T, Tanioka H: Contrastenhanced MR images in patients with meningioma: Importance of enhancement of the dura adjacent to the tumor. AJNR 11: 935-938, 1990

2) Borovich B, Doron $Y$ : Recurrence of intracranial meningiomas: The role played by regional multicentricity. J Neurosurg 64: 58-63, 1986

3) Borovich B, Doron $Y$, Braun J, Guilburd' JN, Zaaroor M, Goldsher D, Lemberger A, Gruszkiewicz $\mathrm{J}$, Feinsod M: Recurrence of intracranial meningiomas: The role played by regional multicentricity. Part 2: Clinical and radiological aspects. $J$ Neurosurg 65: 168-171, 1986

4) Goldsher D, Litt AW, Pinto RS, Bannon KR, Kricheff II: Dural "tail" associated with meningiomas on Gd-DTPA-enhanced MR images: Characteristics, differential diagnostic value, and possible implications for treatment. Radiology 176: 447-450, 1990

5) Larson JJ, Tew JM Jr, Wiot JG, de Courten-Myers GM: Association of meningiomas with dural "tails": Surgical significance. Acta Neurochir (Wien) 114: 5963,1992

6) Mirimanoff RO, Dosoretz DE, Linggood RM, Ojemann RG, Martuza RL: Meningioma: Analysis of recurrence and progression following neurosurgical resection. $J$ Neurosurg 62: 18-24, 1985

7) $\mathrm{Ng} \mathrm{HK}$, Tse $\mathrm{CCH}$, Lo STH: Meningiomas and arachnoid cells: An immunohistochemical study of epithelial markers. Pathology 19: 253-257, 1987

8) Nomura M, Hasegawa M, Yamashima T, Yamashita J, Suzuki M: Meningeal enhancement on MRI after 
craniotomy. CT Kenkyu 13: 421-428, 1991 (in Japanese)

9) Schnitt SJ, Vogel H: Meningiomas. Diagnostic value of immunoperoxidase staining for epithelial membrane antigen. Am J Surg Pathol 10: 640-649, 1986

10) Schörner $W$, Schubeus $P$, Henkes $H$, Lanksch $W$, Felix R: “Meningeal sign": A characteristic finding of meningiomas on contrast-enhanced MR images. Neuroradiology 32: 90-93, 1990

11) Schwechheimer K, Kartenbeck J, Moll R, Franke WW: Vimentin filament-desmosome cytoskeleton of diverse types of human meningiomas: A distinctive diagnostic feature. Lab Invest 51: 584-591, 1984

12) Simpson D: The recurrence of intracranial meningiomas after surgical treatment. $J$ Neurol Neurosurg Psychiat 20: 22-39, 1957

13) Theaker JM, Gatter KC, Esiri MM, Fleming KA: Epithelial membrane antigen and cytokeratin expression by meningiomas: An immunohistological study. $J$ Clin Pathol 39: 435-439, 1986
14) Tien RD, Yang PJ, Chu PK: "Dural tail sign": A specific MR sign for meningioma? J Comput Assist Tomogr 15: 64-66, 1991

15) Tokumaru A, O'uchi T, Eguchi T, Kawamoto S, Kokubu T, Suzuki M, Kameda T: Prominent meningeal enhancement adjacent to meningioma on Gd-DTPA-enhanced MR images: Histopathologic correlation. Radiology 175: 431-433, 1990

16) Wilms G, Lammens M, Marchal G, Calenbergh FV, Plets C, Fraeyenhoven LV, Baert AL: Thickening of dura surrounding meningioma: MR features. $J$ Comput Assist Tomogr 13: 763-768, 1989

Address reprint requests to: K. Sakai, M.D., Department of Neurosurgery, Shinshu University School of Medicine, 3-1-1 Asahi, Matsumoto, Nagano 390, Japan. 